Supporting Information for:

\title{
Exploring the oxidations states of neptunium with Schiff base coordination complexes
}

Bonnie E. Klamm, ${ }^{\mathrm{a}, \mathrm{b}, \uparrow}$ Cory J. Windorff, ${ }^{\mathrm{a}, \uparrow}$ Cristian Celis-Barros, ${ }^{\mathrm{a}}$ Maria J. Beltran-Leiva, ${ }^{\mathrm{a}}$ Joseph M. Sperling, ${ }^{a}$ Thomas E. Albrecht-Schönzart, ${ }^{\mathrm{a}^{*}}$

${ }^{a}$ Department of Chemistry and Biochemistry, Florida State University, 95 Chieftan Way, RM. 118 DLC, Tallahassee, Florida 32306, United States

bLos Alamos National Laboratory, Los Alamos, New Mexico 87544, United States

These authors contributed equally

*To whom correspondence should be addressed: talbrechtschoenzart@gmail.com

Table of Contents

Page

UV/vis/NIR Spectroscopy.

NMR Spectroscopy. .S6-S8

Cyclic Voltammetry. S9-S10

Theoretical Details $. S 11-S 13$

Crystallographic Details

S14-S15

References S16 


\section{UV/vis/NIR Spectroscopy and Photographs of Compounds}

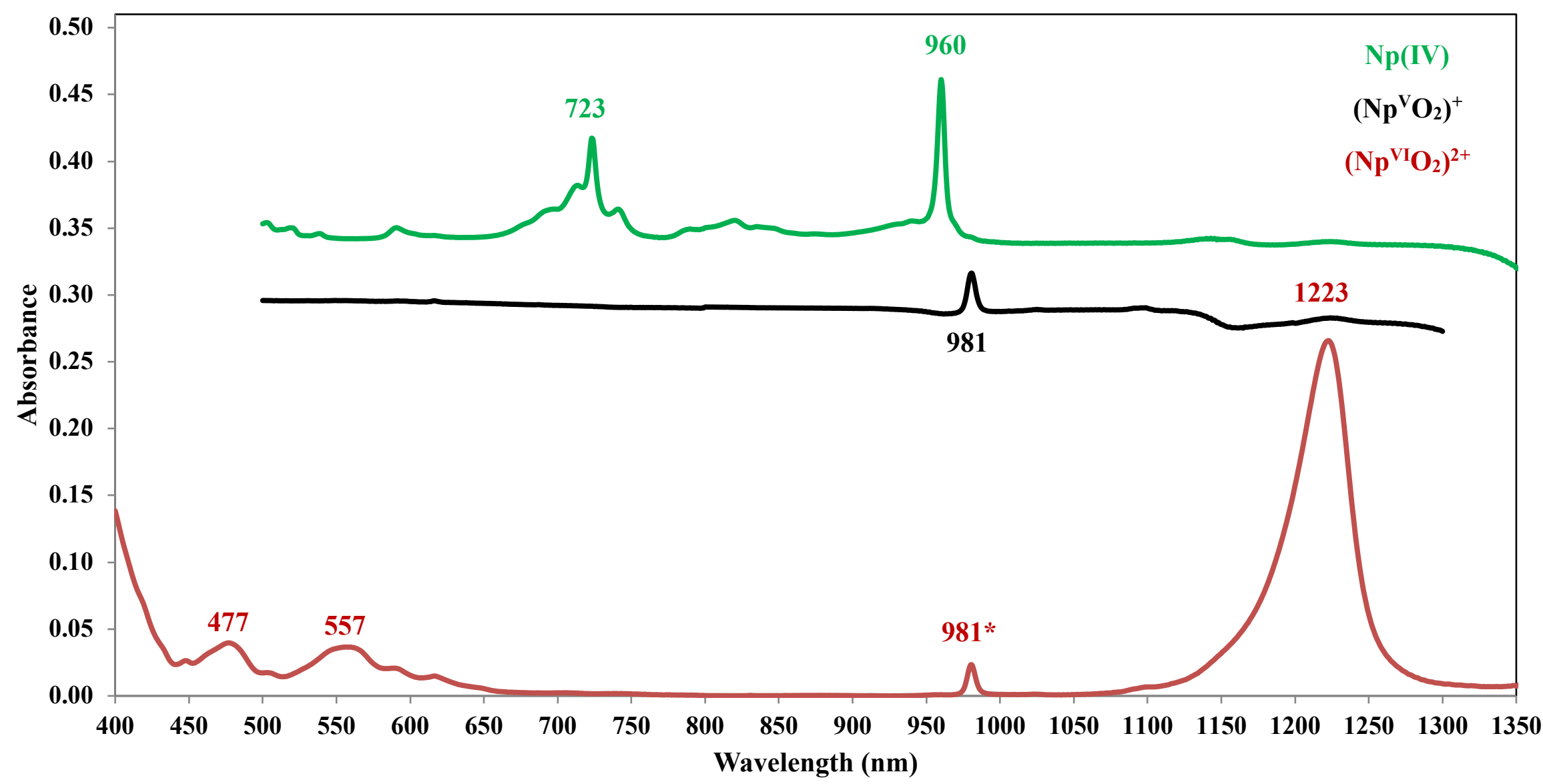

Figure S1. Solution phase UV/vis/NIR of $\mathrm{Np}^{\mathrm{IV}}\left(\mathrm{NO}_{3}\right)_{4}\left(\mathrm{H}_{2} \mathrm{O}\right)_{\times}$(green trace), $\left(\mathrm{Np}^{\mathrm{V}} \mathrm{O}_{2}\right)\left(\mathrm{NO}_{3}\right)\left(\mathrm{H}_{2} \mathrm{O}\right)_{\times}$(black trace), and $\left(\mathrm{Np}^{\mathrm{VI}} \mathrm{O}_{2}\right)\left(\mathrm{NO}_{3}\right)_{2}\left(\mathrm{H}_{2} \mathrm{O}\right)_{x}$ (red trace) in $1 \mathrm{M} \mathrm{HClO}_{4}$. *indicates a small $\left(\mathrm{Np}^{\mathrm{V}} \mathrm{O}_{2}\right)^{+}$impurity. (Note that spectra were recorded on different concentrations from each other with the intent to prove oxidation state purity and therefore the relative intensities are not representative of the actual intensities. 


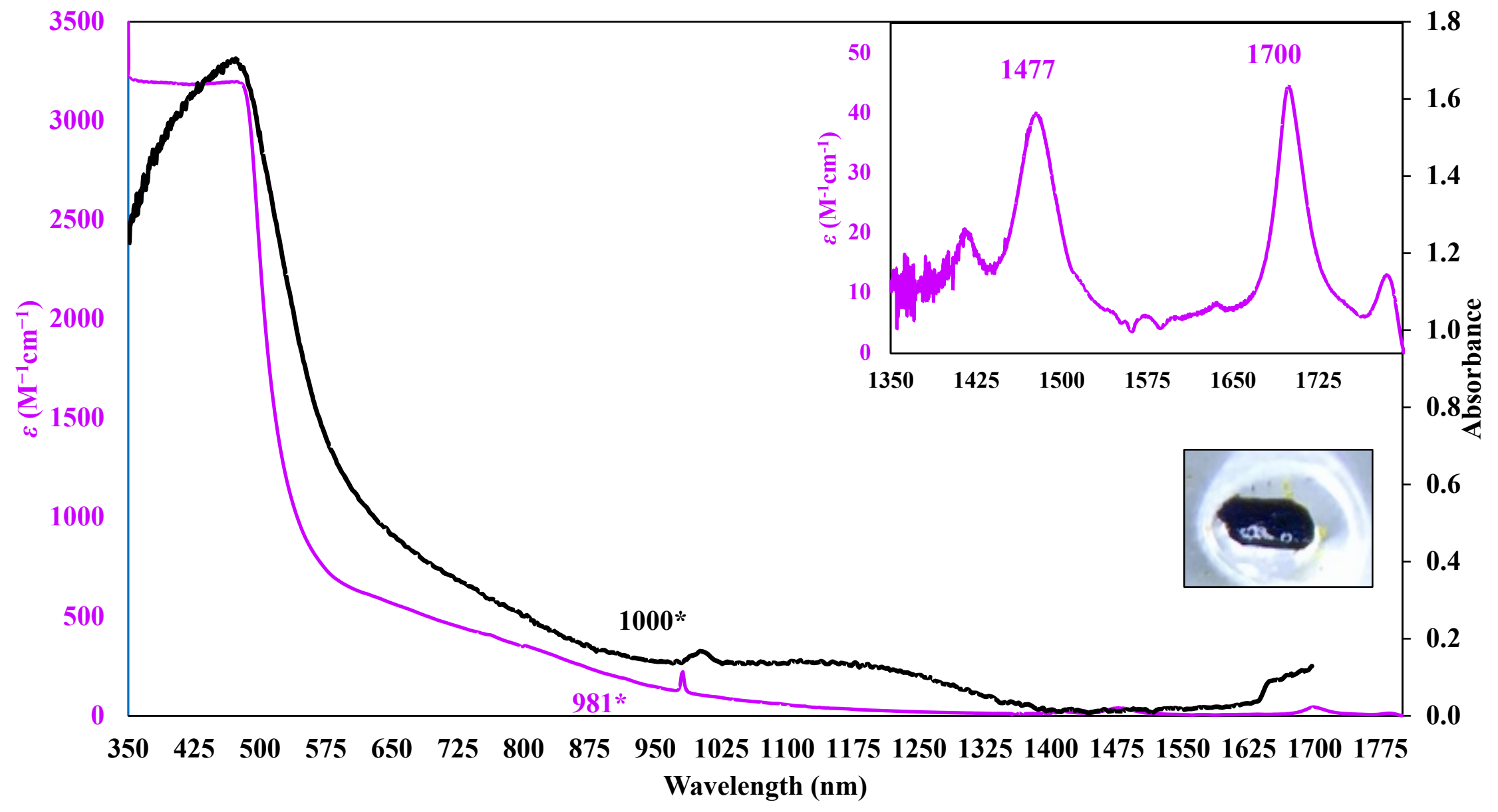

Figure S2. UV/vis/NIR absorption spectrum of $\mathbf{N p}^{\mathrm{VI}} \mathbf{O}_{2} \mathbf{L}(\mathbf{M e O H})$ in the solution phase (purple trace, DMSO- $d_{6}$, left axis) and the solid state (black trace, right axis) with inset to show weak solution phase excitations that are not observed in the solid state, recorded at ambient temperature along with photograph of crystal.* denotes an $\mathrm{Np}(\mathrm{V})$ impurity. 


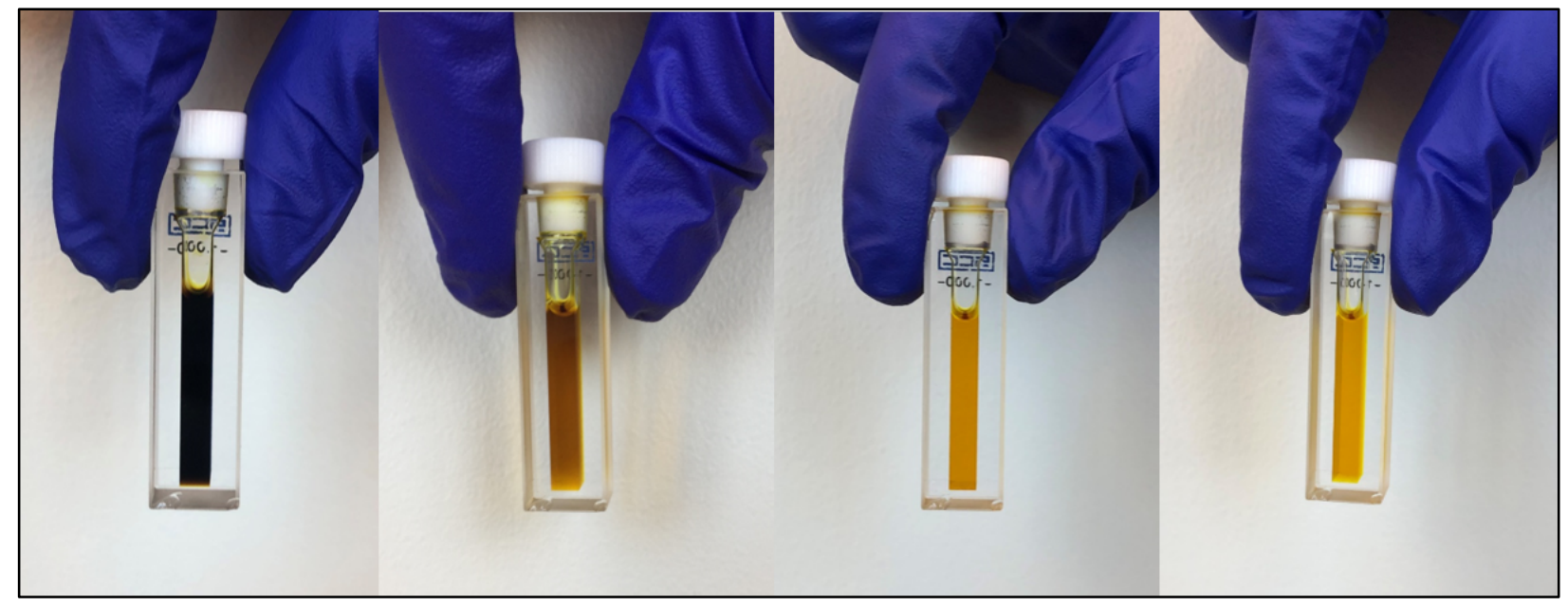

Figure S3. Photographs of $4.75 \mathrm{mM} \mathrm{N} \mathbf{p}^{\mathrm{VI}} \mathbf{O}_{2} \mathbf{L}(\mathbf{M e O H})$ in DMSO- $d_{6}$ (left) with excess additions of $\mathrm{NaNO}_{2}$ and $\mathrm{NH}_{2} \mathrm{OH}$, showing the reduction of $\left(\mathrm{Np}^{\mathrm{VI}} \mathrm{O}_{2}\right)^{2+}$ to $\left(\mathrm{Np}^{\mathrm{V}} \mathrm{O}_{2}\right)^{+}$in solution over time (progressing right).

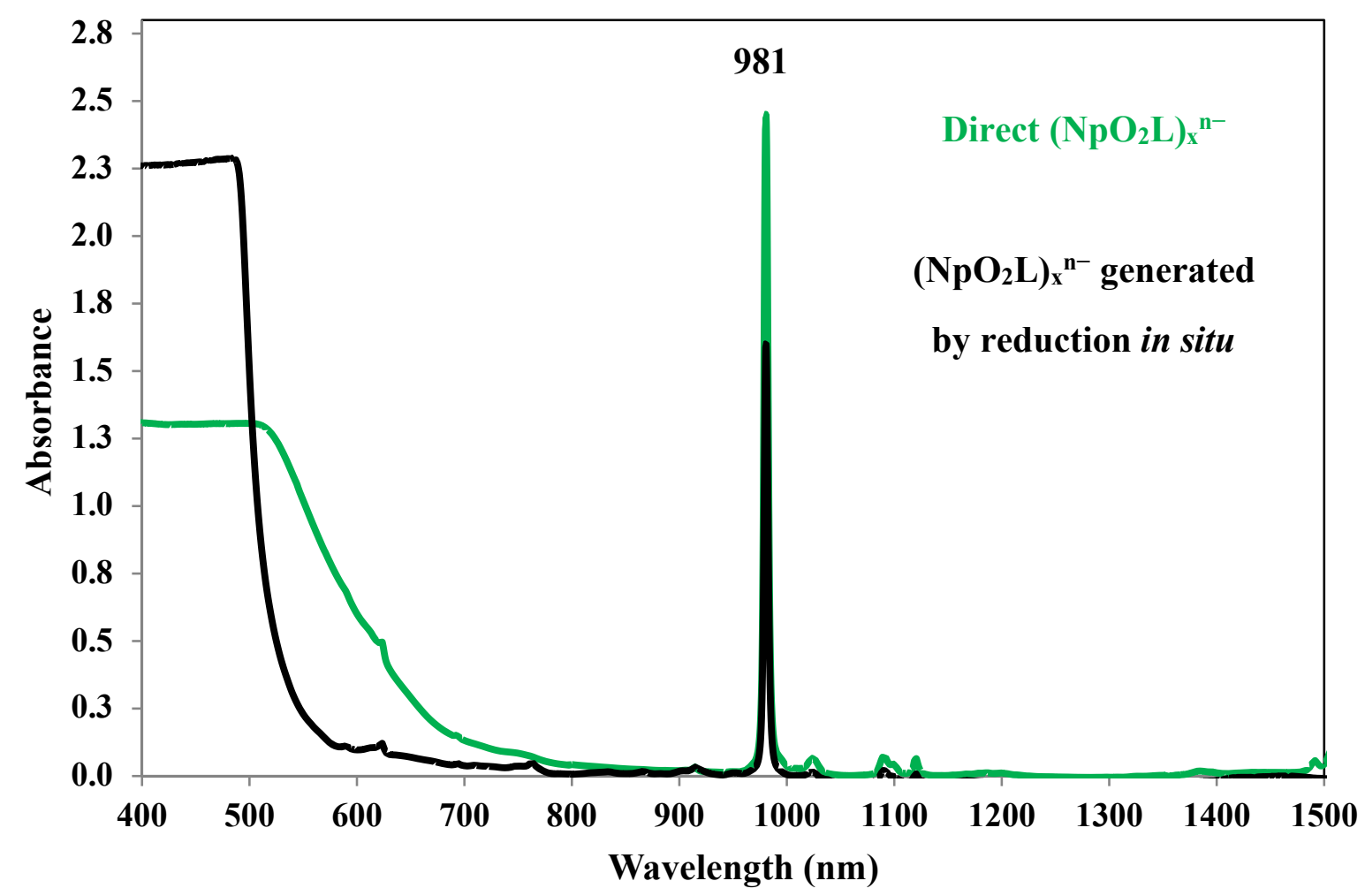

Figure S4. Solution phase UV/vis/NIR of $\left(\mathbf{N p}^{\mathrm{V}} \mathbf{O}_{2} \mathbf{L}\right)_{\mathbf{x}}{ }^{\mathbf{n}-}$ from a direct synthesis (green trace) compared with spectrum of $\mathbf{N p}^{\mathbf{V I}} \mathbf{O}_{2} \mathbf{L}$ after treatment with $\mathrm{NaNO}_{2}$ and $\mathrm{H}_{2} \mathrm{NOH} \cdot \mathrm{HCl}$ (black trace) in $\mathrm{DMSO}-d_{6}$ at ambient temperature. 


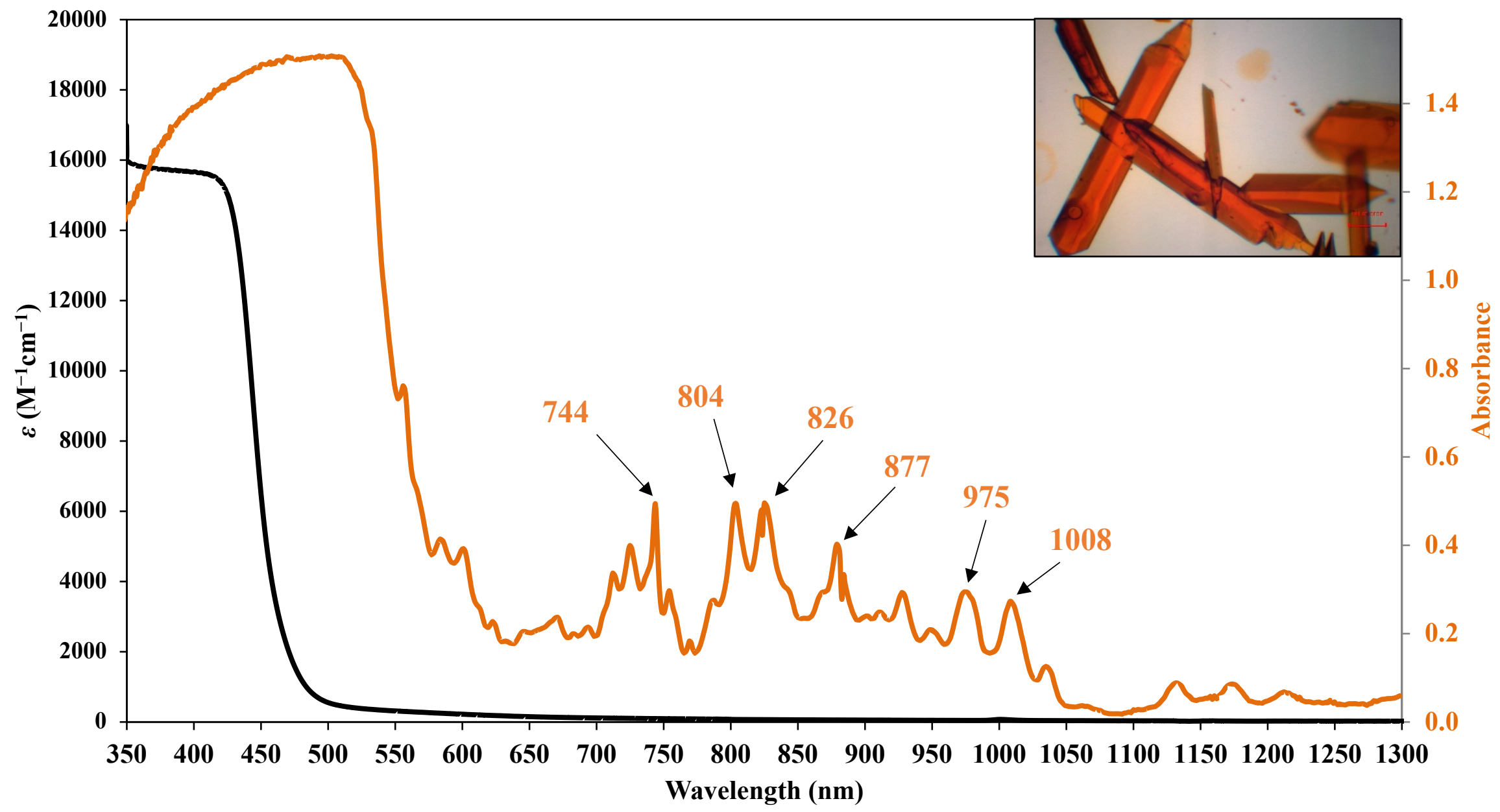

Figure S5. UV/vis/NIR absorption spectrum of $\mathbf{N p}^{\mathrm{IV}} \mathbf{L}_{2}$ in the solution phase (black trace, toluene, left axis) and the solid state (orange trace, right axis), recorded at ambient temperature along with photograph of crystals. 


\section{NMR Spectroscopy}

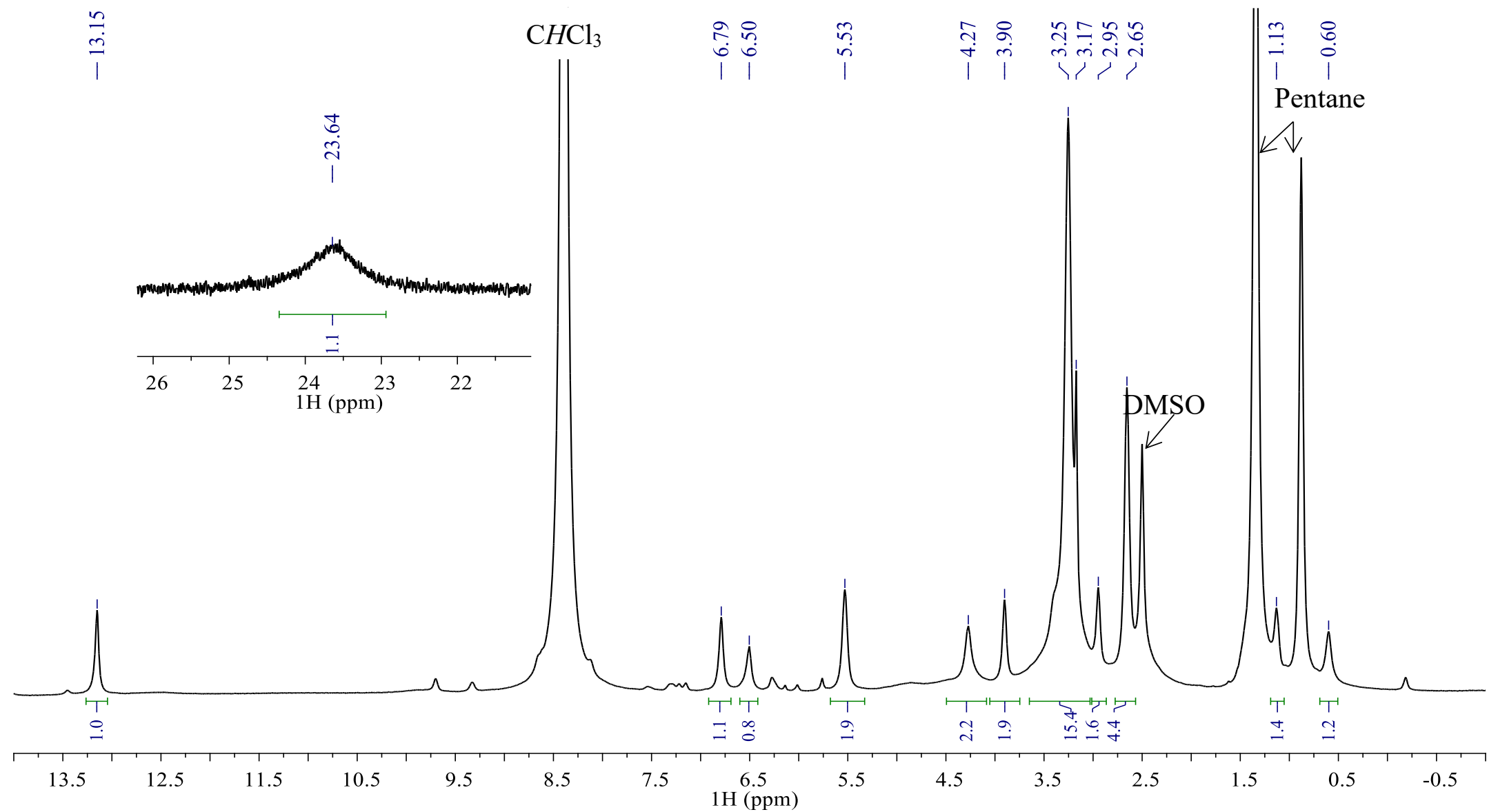

Figure S6. ${ }^{1} \mathrm{H}$ NMR of $\mathrm{Np}^{\mathrm{VI}} \mathbf{O}_{2} \mathbf{L}(\mathbf{M e O H})$, inset shows additional downfield resonance, in DMSO- $d_{6}$ at room temperature. There is a large $\mathrm{CHCl}_{3}$ peak from an unsuccessful Evans' method attempt. 


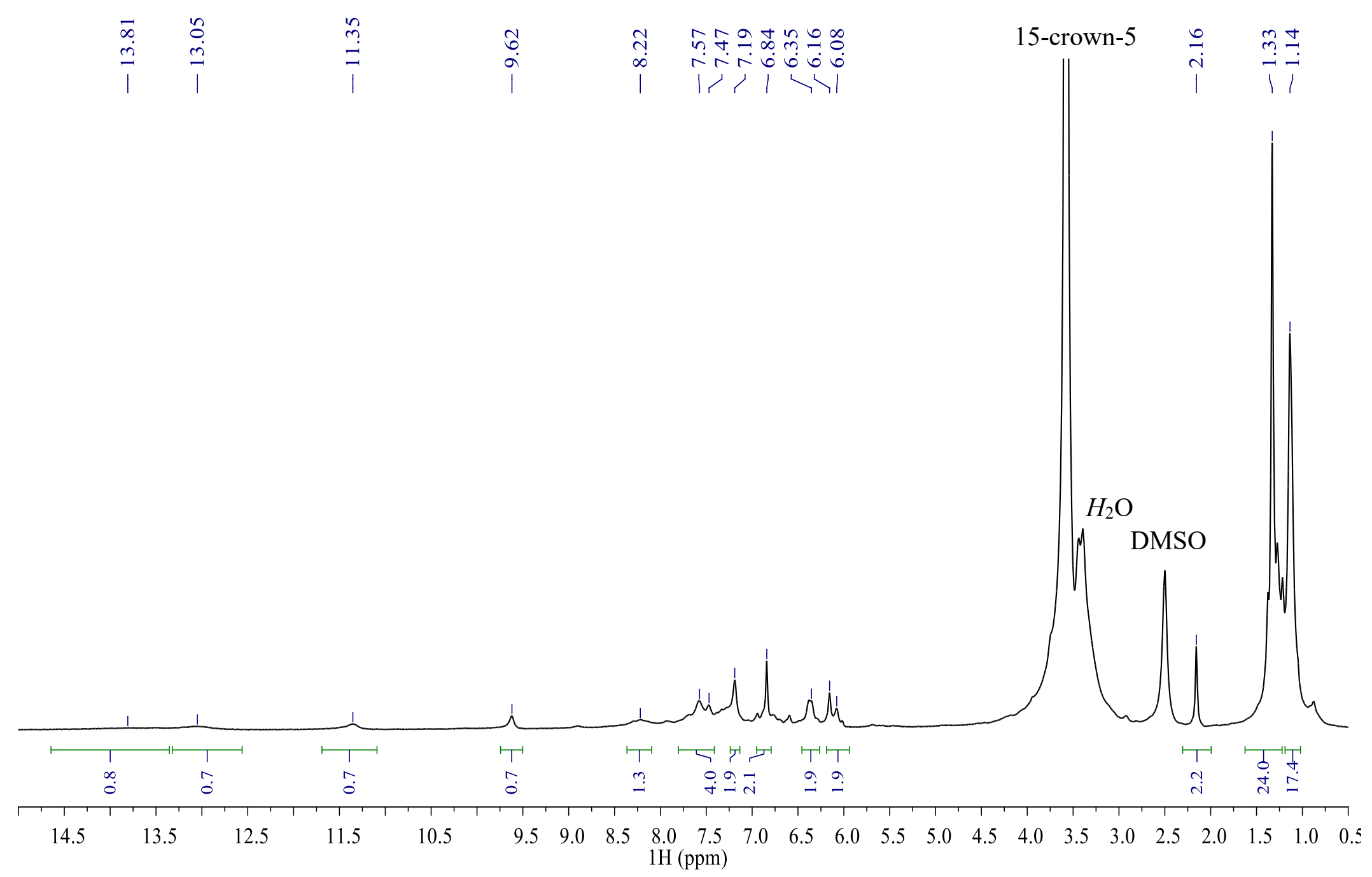

Figure S7. ${ }^{1} \mathrm{H}$ NMR of $\left(\mathbf{N p}^{\mathbf{V}} \mathbf{O}_{2} \mathbf{L}\right)_{\mathbf{x}}{ }^{\mathbf{n}-}$ in DMSO- $d_{6}$ at room temperature, integrations are tentative. 


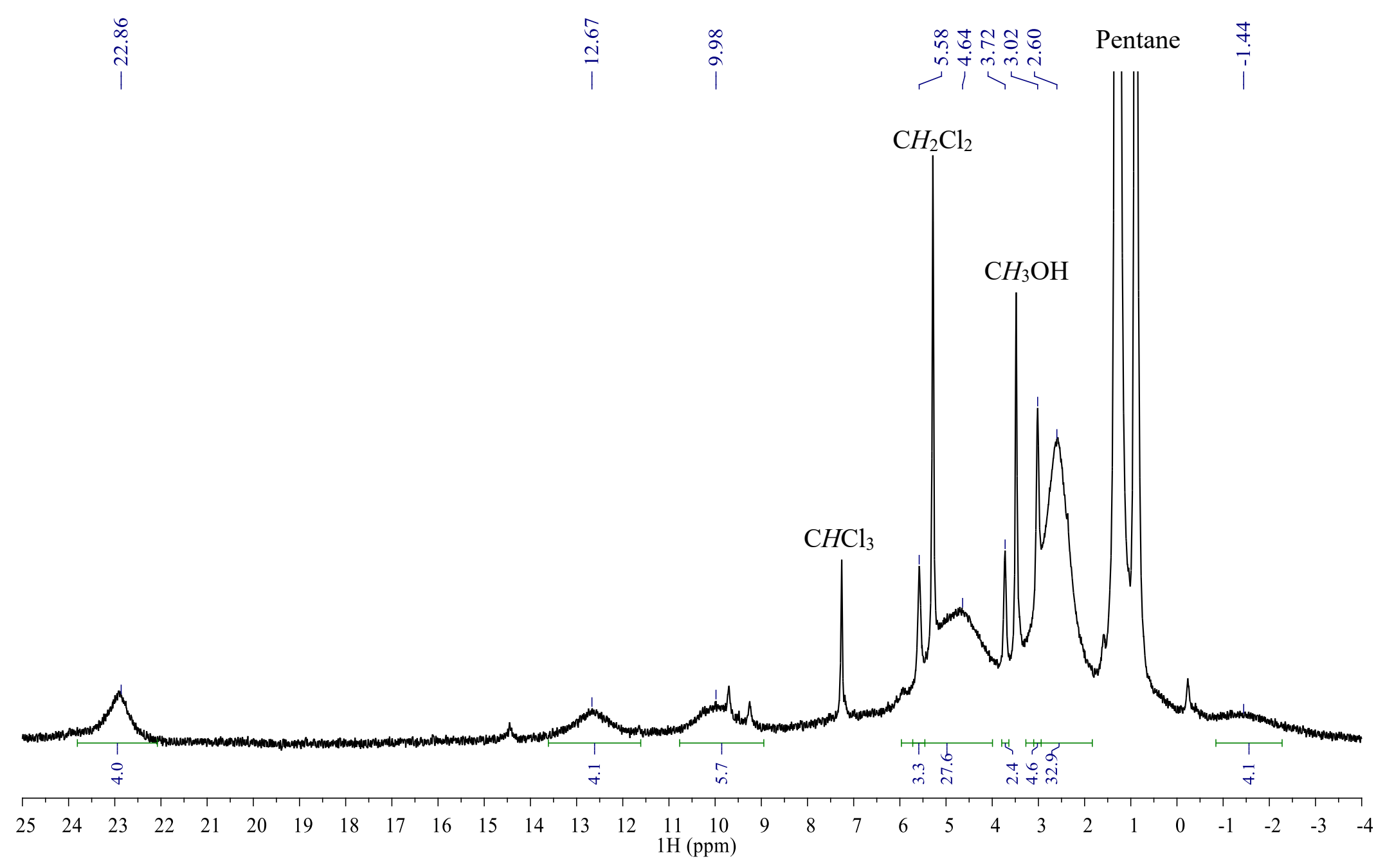

Figure S8. ${ }^{1} \mathrm{H}$ NMR of $\mathbf{N p}^{\mathrm{IV}} \mathbf{L}_{2}$ in $\mathrm{CDCl}_{3}$ at room temperature. 


\section{Cyclic Voltammetry}

The diffusion coefficient (D) for $\mathbf{N p}^{\mathrm{VI}} \mathbf{O}_{2} \mathbf{L}(\mathbf{M e O H})$ were calculated using the Randles-Sevcik equation, Eq.1,${ }^{1}$ by plotting the peak current $\left(i_{p}\right)$ vs. the square root of the scan rate $\left(v^{1 / 2}\right)$; where $n, A$, and $C^{0}$, are the electron stoichiometry in the rate determining process, surface area of the working electrode, and concentration of the oxidant, respectively.

$$
i_{p}=0.4463 n F A C^{0}\left(\frac{\mathrm{nFvD}}{\mathrm{RT}}\right)^{1 / 2}
$$

Estimating the heterogeneous electron transfer constant, $k^{0}$ is of primary interest when the performance of electrode materials are examined. This allows for an indication of the speed of electron transfer between an electroactive species and an electrode surface. The simplified Nicholson method used here ${ }^{2,3}$ utilizes the link between the Nicholson parameter and $k^{0}$ by investigating the peak to peak separation $\left(\Delta E_{p}\right)$, Eq. 2; which allows for the estimation of the heterogeneous electron transfer rate constant $\left(k^{0}\right), \mathbf{E q} 3$.

$$
\begin{gathered}
\Psi=\frac{-0.6288+0.0021\left(\Delta E_{p}\right)}{1-0.017\left(\Delta E_{p}\right)} \\
\Psi=\mathrm{k}^{0}\left(\frac{\pi D n F v}{R T}\right)^{-1 / 2}
\end{gathered}
$$

Through mathematical manipulation, $k^{0}$ is taken directly from the slope of the graph of $\psi$ vs. $\left(\frac{\pi D F v}{R T}\right)^{-1 / 2}$, Table S1. This method only works for quasi reversible systems, since it relies 
upon the fact that as the $\Delta E_{p}$ increases, the scan rate increases because the rate of mass transport becomes quicker or equal in rate as the electron transfer.

Table S1. Kinetic data for $\mathrm{Np}^{\mathrm{VI}} \mathrm{O}_{2} \mathrm{~L}(\mathrm{MeOH})$.

\begin{tabular}{ccc}
\hline $\begin{array}{c}\text { Scan Rate } \\
(\mathrm{V} / \mathrm{s})\end{array}$ & $\begin{array}{c}\text { Reversibility } \\
\Delta E_{p}(\mathrm{mV})\end{array}$ & $\begin{array}{c}\text { Nicholson } \\
\text { Parameter }(\Psi)\end{array}$ \\
\hline 0.025 & 91 & 0.80 \\
0.05 & 103 & 0.55 \\
0.1 & 117 & 0.39 \\
0.2 & 136 & 0.26 \\
0.3 & 148 & 0.21 \\
0.4 & 158 & 0.18
\end{tabular}

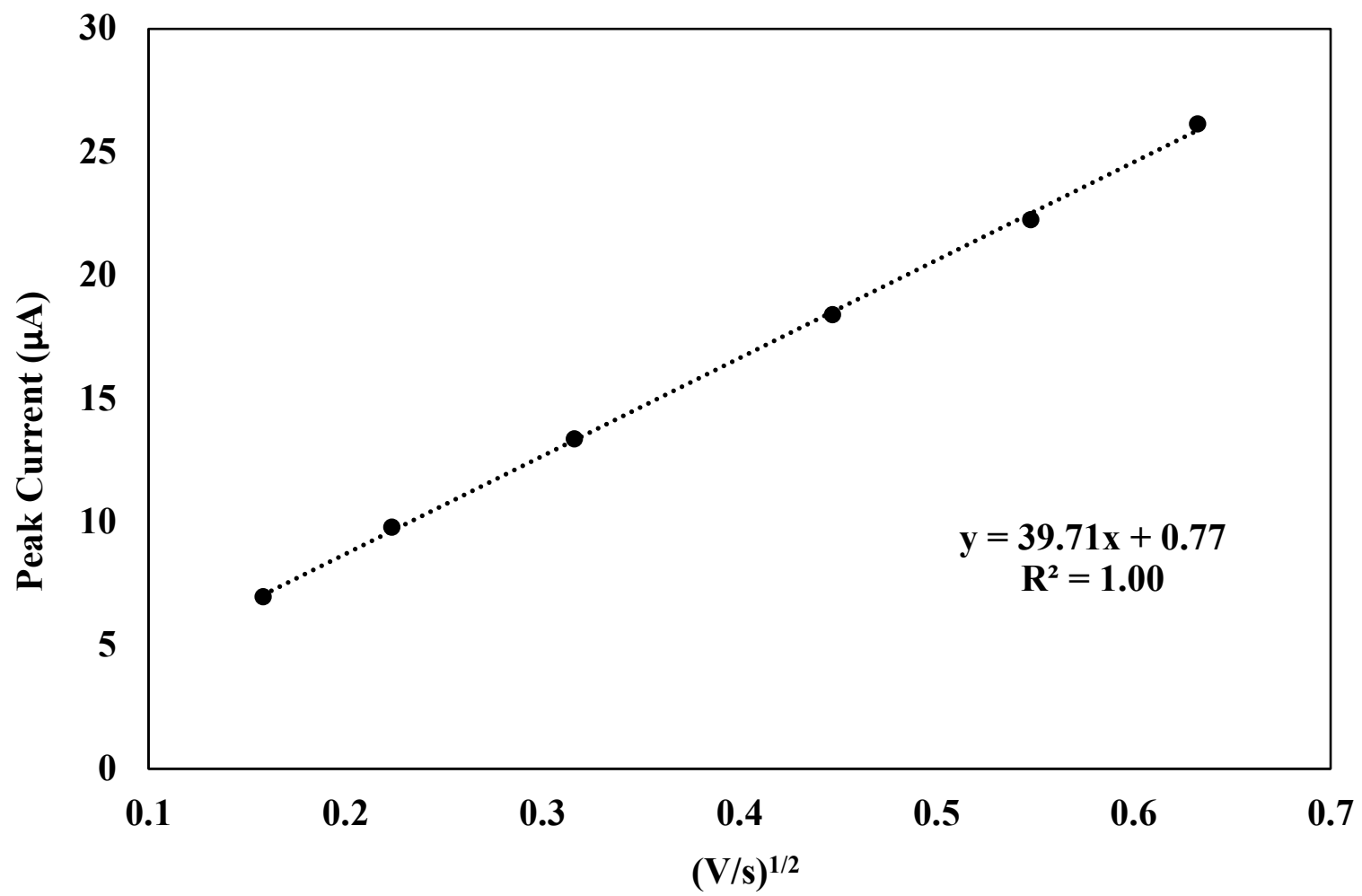

Figure S9. A plot of the (scan rate) $)^{1 / 2}$ and value of cathodic peak currents show a linear relationship, allowing a calculation of $\mathrm{D}=2.18 \times 10^{-8} \mathrm{~cm}^{2} / \mathrm{s}$ for $\mathbf{N p}^{\mathrm{VI}} \mathbf{O}_{2} \mathbf{L}(\mathbf{M e O H})$. 


\section{Theoretical Details}

Table S2. Decomposition of the multiconfigurational ground and first excited states using the SO-CASSCF/PT2 $(6,10)$ wave functions. Ligand orbitals $p_{\sigma}$ and $p_{\pi}$ are depicted in red and refer to the mixing of the $2 p$ axial oxygen orbitals with $5 f$ orbitals.

\begin{tabular}{|c|c|c|c|c|c|}
\hline SO-PT2 State & \multicolumn{2}{|c|}{$\left(\mathrm{Np}^{\mathrm{VI}} \mathrm{O}_{2}\right)^{2+}$} & SO-PT2 State & \multicolumn{2}{|c|}{$\mathrm{Np}^{\mathrm{VI}} \mathrm{O}_{2} \mathrm{~L}(\mathrm{MeOH})$} \\
\hline \multirow{10}{*}{${ }^{2} \Phi_{5 / 2 \mathrm{u}}$} & $82 \%$ & $p_{\pi}^{4} p_{\sigma}^{2} f_{\delta}^{0}$ & \multirow{10}{*}{${ }^{2} \Phi_{5 / 2 \mathrm{u}}$} & $61 \%$ & $p_{\pi}^{4} p_{\sigma^{2}} f_{\delta}^{0} f_{\varphi}{ }^{1} f_{\pi^{0}}^{0} f_{\sigma}^{0}$ \\
\hline & & $f_{\varphi}^{1} f_{\pi}^{0} f_{\sigma}^{0}$ & & & \\
\hline & $8 \%$ & $p_{\pi}^{3} p_{\sigma}^{2} f_{\delta}^{0}$ & & $21 \%$ & $p_{\pi}^{3} p_{\sigma}^{2} f_{\delta}^{0} f_{\varphi}^{1} f_{\pi}^{1} f_{\sigma}^{0}$ \\
\hline & & $f_{\varphi}^{1} f_{\pi}^{1} f_{\sigma}^{0}$ & & & \\
\hline & $2 \%$ & $p_{\pi}^{3} p_{\sigma}^{2} f_{\delta}^{0}$ & & $3 \%$ & $p_{\pi}^{4} p_{\sigma}{ }^{1} f_{\delta}^{0} f_{\varphi}{ }^{1} f_{\pi}{ }^{0} f_{\sigma}{ }^{1}$ \\
\hline & & $f_{\varphi}^{1} f_{\pi}^{1} f_{\sigma}^{0}$ & & & \\
\hline & $2 \%$ & $p_{\pi}^{4} p_{\sigma}^{1} f_{\delta}^{0}$ & & $3 \%$ & $p_{\pi}^{4} p_{\sigma^{2}} f_{\delta}{ }^{1} f_{\varphi}^{0} f_{\pi}^{0} f_{\sigma}^{0}$ \\
\hline & & $f_{\varphi}^{1} f_{\pi}^{0} f_{\sigma}^{1}$ & & & \\
\hline & $1 \%$ & $p_{\pi}^{3} p_{\sigma}^{1} f_{\delta}^{0}$ & & $2 \%$ & $p_{\pi}^{3} p_{\sigma}^{2} f_{\delta}^{0} f_{\varphi}^{2} f_{\pi}^{0} f_{\sigma}^{0}$ \\
\hline & & $f_{\varphi}^{1} f_{\pi}^{1} f_{\sigma}^{1}$ & & & \\
\hline & $82 \%$ & $p_{\pi}^{4} p_{\sigma}^{2} f_{\delta}^{1}$ & & $64 \%$ & $p_{\pi}^{4} p_{\sigma}^{2} f_{\delta}{ }^{1} f_{\varphi}{ }^{0} f_{\pi}{ }^{0} f_{\sigma}^{0}$ \\
\hline & & $f_{\varphi}^{0} f_{\pi}^{0} f_{\sigma}^{0}$ & & & \\
\hline & $9 \%$ & $p_{\pi}^{3} p_{\sigma}^{2} f_{\delta}^{1}$ & & $21 \%$ & $p_{\pi}^{3} p_{\sigma^{2}}^{2} f_{\delta}^{1} f_{\varphi}^{0} f_{\pi}^{1} f_{\sigma}^{0}$ \\
\hline & & $f_{\varphi}^{0} f_{\pi}^{1} f_{\sigma}^{0}$ & & & \\
\hline 21 & $2 \%$ & $p_{\pi}^{4} p_{\sigma}^{1} f_{\delta}^{1}$ & 21 & $3 \%$ & $p_{\pi}^{4} p_{\sigma}^{1} f_{\delta}^{1} f_{\varphi}^{0} f_{\pi}^{0} f_{\sigma}^{1}$ \\
\hline${ }^{2} \Delta / 2 \mathrm{u}$ & & $f_{\varphi}^{0} f_{\pi}^{0} f_{\sigma}^{1}$ & ${ }^{2} \Delta_{3 / 2 \mathrm{u}}$ & & \\
\hline & $2 \%$ & $p_{\pi}^{3} p_{\sigma}^{1} f_{\delta}^{1}$ & & $2 \%$ & $p_{\pi}^{3} p_{\sigma}^{2} f_{\delta}{ }^{1} f_{\varphi}^{0} f_{\pi}^{1} f_{\sigma}^{0}$ \\
\hline & & $f_{\varphi}^{0} f_{\pi}^{1} f_{\sigma}^{1}$ & & & \\
\hline & $1 \%$ & $p_{\pi}^{2} p_{\sigma}^{2} f_{\delta}^{1}$ & & $1 \%$ & $p_{\pi}^{4} p_{\sigma}^{0} f_{\delta}^{1} f_{\varphi}^{0} f_{\pi}^{0} f_{\sigma}^{2}$ \\
\hline & & $f_{\varphi}^{0} f_{\pi}^{2} f_{\sigma}^{0}$ & & & \\
\hline
\end{tabular}


Table S3. QTAIM metrics derived from CASSCF wavefunctions. Electron densities $\rho(\mathrm{r})$ are given in e Bohr ${ }^{-3}$; potential $\mathrm{V}(\mathrm{r})$, kinetic $\mathrm{G}(\mathrm{r})$, and total $\mathrm{H}(\mathrm{r})$ energy densities in $\mathrm{kJ} \mathrm{mol}^{-1} \mathrm{Bohr}^{-3}$.

\begin{tabular}{|c|c|c|c|c|c|c|c|c|}
\hline \multirow{4}{*}{$\left(\mathrm{Np}^{\mathrm{VI}} \mathrm{O}_{2}\right)^{2+}$} & Np-X & $\rho(\mathbf{r})$ & $\delta(\mathbf{r})$ & $V(r)$ & G(r) & $H(r)$ & $\mathbf{V}(\mathbf{r}) / \mathbf{G}(\mathbf{r})$ & $\mathrm{H}(\mathrm{r}) / \boldsymbol{\rho}(\mathbf{r})$ \\
\hline & $\mathrm{O}_{\mathrm{yl}}(1)$ & 0.3383 & 1.5134 & -2076.6 & 1148.4 & -928.2 & 1.81 & -2743.7 \\
\hline & $\mathrm{O}_{\mathrm{yl}}(2)$ & 0.3386 & 1.5202 & -2077.4 & 1147.4 & -930.0 & 1.81 & -2746.7 \\
\hline & Total & 0.6769 & 3.0336 & -4154.0 & 2295.8 & -1858.2 & 1.81 & -5490.4 \\
\hline \multirow[t]{8}{*}{$\mathrm{Np}^{\mathrm{VI}} \mathrm{O}_{2} \mathrm{~L}_{2}(\mathrm{MeOH})$} & $\mathrm{O}_{\mathrm{yl}}(1)$ & 0.2842 & 0.9294 & -1797.6 & 1143.7 & -653.9 & 1.57 & -2300.8 \\
\hline & $\mathrm{O}_{\mathrm{yl}}(2)$ & 0.2628 & 1.0496 & -1572.7 & 995.0 & -577.7 & 1.58 & -2198.3 \\
\hline & $\mathrm{N}_{\mathrm{L}}(1)$ & 0.0523 & 0.2400 & -143.0 & 130.3 & -12.7 & 1.10 & -243.6 \\
\hline & $\mathrm{N}_{\mathrm{L}}(2)$ & 0.0562 & 0.2508 & -157.8 & 142.0 & -15.9 & 1.11 & -282.2 \\
\hline & $\mathrm{O}_{\mathrm{L}}(1)$ & 0.0762 & 0.3256 & -272.0 & 242.1 & -29.9 & 1.12 & -392.4 \\
\hline & $\mathrm{O}_{\mathrm{L}}(2)$ & 0.0909 & 0.3818 & -351.5 & 304.7 & -46.8 & 1.15 & -514.9 \\
\hline & $\mathrm{O}_{\mathrm{MeOH}}$ & 0.0528 & 0.2131 & -169.5 & 164.1 & -5.5 & 1.03 & -103.4 \\
\hline & Total & 0.8754 & 3.3903 & -4464.2 & 3121.8 & -1342.4 & 1.43 & -6035.6 \\
\hline \multirow[t]{5}{*}{$\mathbf{N} \mathbf{p}^{\mathrm{IV}} \mathbf{L}_{2}$} & $\mathrm{O}_{\mathrm{L}}(1)$ & 0.0873 & 0.3915 & -338.0 & 292.2 & -45.8 & 1.16 & -524.2 \\
\hline & $\mathrm{O}_{\mathrm{L}}(2)$ & 0.0900 & 0.3876 & -323.4 & 280.0 & -43.4 & 1.16 & -482.4 \\
\hline & $\mathrm{N}_{\mathrm{L}}(1)$ & 0.0500 & 0.2300 & -130.5 & 118.6 & -12.0 & 1.10 & -239.2 \\
\hline & $\mathrm{N}_{\mathrm{L}}(2)$ & 0.0524 & 0.2313 & -138.6 & 124.8 & -13.8 & 1.11 & -263.0 \\
\hline & Total & 0.5594 & 2.4805 & -1861.1 & 1631.2 & -229.8 & 1.14 & -3017.6 \\
\hline
\end{tabular}



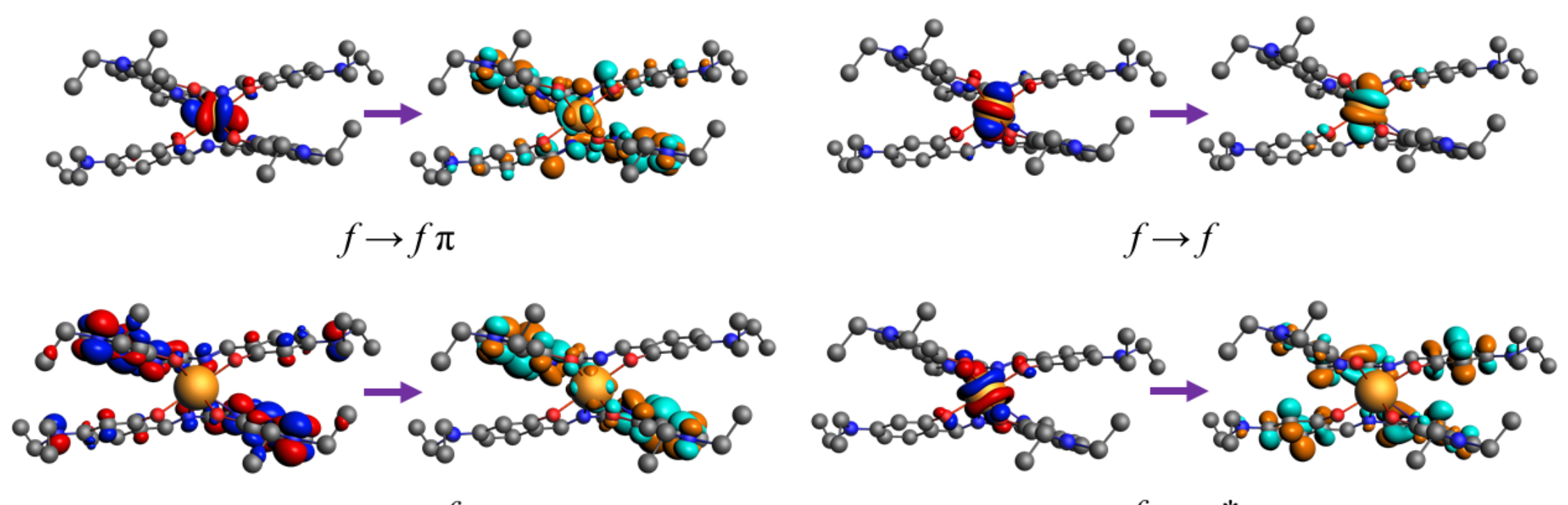

$$
\pi \rightarrow \pi f \quad f \rightarrow \pi^{*}
$$

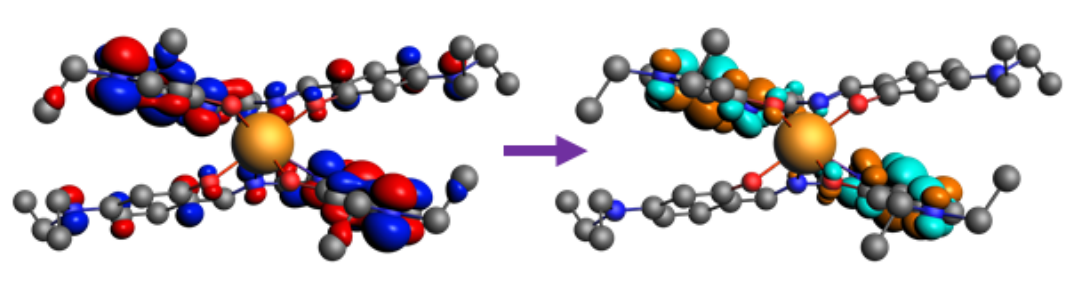

$$
\pi \rightarrow \pi^{*}
$$

Figure S10. Selected natural transition orbitals (NTOs) obtained from TD-DFT representing the main transitions involved in the absorption spectrum of $\mathbf{N} \mathbf{p}^{\mathbf{I V}} \mathbf{L}_{2}$. Blue/red and green/orange NTOs correspond to donors and acceptors, respectively. 


\section{Crystallographic Details}

Table S4. Crystallographic information for $\mathrm{Np}^{\mathrm{IV}} \mathrm{L}_{2}$ and $\mathrm{Np}^{\mathrm{VI}} \mathrm{O}_{2} \mathrm{~L}(\mathrm{MeOH})$

\begin{tabular}{|c|c|c|}
\hline Compound & $N p^{I V} L_{2} \cdot 2 D C M$ & $\mathrm{~Np}^{\mathrm{VI}} \mathrm{O}_{2} \mathrm{~L}(\mathrm{MeOH})$ \\
\hline Empirical Formula & $\mathrm{C}_{58} \mathrm{H}_{68} \mathrm{~N}_{8} \mathrm{O}_{4} \mathrm{Cl}_{4} \mathrm{~Np}$ & $\mathrm{C}_{29} \mathrm{H}_{36} \mathrm{~N}_{4} \mathrm{O}_{5} \mathrm{~Np}$ \\
\hline Color & Orange & Black \\
\hline Habit & Columnar & Irregular \\
\hline Temperature (K) & $298(2)$ & 100 \\
\hline Crystal System & Monoclinic & Monoclinic \\
\hline Space Group & $C 2 / c$ & $P 2_{1} / c$ \\
\hline$a(\AA)$ & 14.9971(12) & $14.4694(6)$ \\
\hline$b(\stackrel{\AA}{A})$ & $24.825(2)$ & $10.0621(4)$ \\
\hline$c(\stackrel{\AA}{)})$ & $15.306(2)$ & $19.2240(8)$ \\
\hline$\alpha\left(^{\circ}\right)$ & 90 & 90 \\
\hline$\beta\left({ }^{\circ}\right)$ & $95.112(2)$ & $99.0190(10)$ \\
\hline$\gamma\left({ }^{\circ}\right)$ & 90 & 90 \\
\hline Volume $\left(\AA^{3}\right)$ & $5675.8(10)$ & $2764.3(2)$ \\
\hline $\mathbf{Z}$ & 4 & 4 \\
\hline$\rho_{\text {calcd }}\left(\mathbf{M g} / \mathbf{m}^{3}\right)$ & 1.545 & 1.820 \\
\hline$\mu\left(\mathbf{m m}^{-1}\right)$ & 2.073 & 3.805 \\
\hline $\mathrm{R}^{a}{ }^{a}(I>2.0 \sigma(\mathrm{I}))$ & 0.0334 & 0.0256 \\
\hline wR2 (all data) & 0.0700 & 0.0608 \\
\hline
\end{tabular}

aDefinitions: $\mathrm{R} 1=\Sigma|| F_{\mathrm{o}}|-| F_{\mathrm{c}}|/ \Sigma| F_{\mathrm{o}} \mid, \mathrm{wR} 2=\left[\Sigma w\left(F_{\mathrm{o}}^{2}-F_{\mathrm{c}}{ }^{2}\right)^{2} / \Sigma w\left(F_{\mathrm{o}}^{2}\right)^{2}\right]^{1 / 2}$.

Goof $=\mathrm{S}=\left[\Sigma\left[\mathrm{w}\left(\mathrm{F}_{\mathrm{o}}{ }^{2}-\mathrm{F}_{\mathrm{c}}{ }^{2}\right)^{2}\right] /(\mathrm{n}-\mathrm{p})\right]^{1 / 2}$ where $\mathrm{n}$ is the number of reflections and $\mathrm{p}$ is the total number of parameters refined. 
Table S5. Bond lengths $[\AA]$ and angles $\left[^{\circ}\right]$ for $\mathbf{N p}^{\mathrm{IV}} \mathbf{L}_{2}$

\begin{tabular}{|c|c|c|c|c|c|c|c|}
\hline Atom & Atom & \multicolumn{2}{|c|}{ Length/Å } & $\mathrm{O} 1$ & $\mathrm{~Np} 1$ & $\mathrm{O} 2$ & $85.37(7)$ \\
\hline $\mathrm{Np} 1$ & $\mathrm{O} 2$ & \multicolumn{2}{|c|}{$2.2378(18)$} & $\mathrm{O} 1^{1}$ & Np1 & $\mathrm{O} 2^{1}$ & $85.37(7)$ \\
\hline Np1 & $\mathrm{O} 2^{1}$ & \multicolumn{2}{|c|}{$2.2378(18)$} & $\mathrm{O} 1^{1}$ & $\mathrm{~Np} 1$ & $\mathrm{O} 2$ & $83.21(7)$ \\
\hline $\mathrm{Np} 1$ & $\mathrm{O} 1$ & \multicolumn{2}{|c|}{$2.2238(19)$} & $\mathrm{O} 1$ & $\mathrm{~Np} 1$ & $\mathrm{O} 2^{1}$ & $83.21(7)$ \\
\hline $\mathrm{Np} 1$ & $\mathrm{O} 1^{1}$ & \multicolumn{2}{|c|}{$2.2238(19)$} & $\mathrm{O} 1^{1}$ & Np1 & $\mathrm{O} 1$ & $164.13(10)$ \\
\hline Np1 & N1 & \multicolumn{2}{|c|}{$2.573(2)$} & $\mathrm{O} 1$ & Np1 & N1 & $70.86(7)$ \\
\hline Np1 & $\mathrm{N} 1^{1}$ & \multicolumn{2}{|c|}{$2.573(2)$} & $\mathrm{O} 1$ & $\mathrm{~Np} 1$ & $\mathrm{~N} 1^{1}$ & $123.71(7)$ \\
\hline $\mathrm{Np} 1$ & $\mathrm{~N} 2$ & \multicolumn{2}{|c|}{$2.550(2)$} & $\mathrm{O} 1^{1}$ & $\mathrm{~Np} 1$ & N1 & $123.71(7)$ \\
\hline \multirow[t]{2}{*}{ Np1 } & \multirow[t]{2}{*}{$\mathrm{N} 2^{1}$} & \multicolumn{2}{|c|}{$2.550(2)$} & $\mathrm{O} 1^{1}$ & Np1 & $\mathrm{N} 1^{1}$ & $70.85(7)$ \\
\hline & & & & $\mathrm{O} 1^{1}$ & $\mathrm{~Np} 1$ & N2 & $74.03(7)$ \\
\hline Atom & Atom & Atom & Angle/ ${ }^{\circ}$ & $\mathrm{O} 1$ & $\mathrm{~Np} 1$ & $\mathrm{~N} 2^{1}$ & $74.03(7)$ \\
\hline $\mathrm{O} 2$ & Np1 & $\mathrm{O} 2^{1}$ & $87.83(10)$ & $\mathrm{O} 1$ & Np1 & $\mathrm{N} 2$ & $112.58(7)$ \\
\hline $\mathrm{O} 2^{1}$ & Np1 & N1 & $146.04(7)$ & $\mathrm{O} 1^{1}$ & $\mathrm{~Np} 1$ & $\mathrm{~N} 2^{1}$ & $112.58(7)$ \\
\hline $\mathrm{O} 2$ & Np1 & $\mathrm{N} 1^{1}$ & $146.04(7)$ & $\mathrm{N} 1^{1}$ & $\mathrm{~Np} 1$ & N1 & $69.41(10)$ \\
\hline $\mathrm{O} 2$ & $\mathrm{~Np} 1$ & N1 & $110.79(7)$ & $\mathrm{N} 2^{1}$ & $\mathrm{~Np} 1$ & $\mathrm{~N} 1^{1}$ & $61.38(7)$ \\
\hline $\mathrm{O} 2^{1}$ & $\mathrm{~Np} 1$ & $\mathrm{~N} 1^{1}$ & $110.79(7)$ & N2 & $\mathrm{Np} 1$ & $\mathrm{~N} 1^{1}$ & $80.29(7)$ \\
\hline $\mathrm{O} 2$ & Np1 & $\mathrm{N} 2$ & $71.54(7)$ & $\mathrm{N} 2^{1}$ & $\mathrm{~Np} 1$ & N1 & $80.29(7)$ \\
\hline $\mathrm{O} 2^{1}$ & Np1 & $\mathrm{N} 2$ & $152.14(7)$ & $\mathrm{N} 2$ & $\mathrm{~Np} 1$ & N1 & $61.38(7)$ \\
\hline $\mathrm{O} 2^{1}$ & Np1 & $\mathrm{N} 2^{1}$ & $71.54(7)$ & $\mathrm{N} 2^{1}$ & $\mathrm{~Np} 1$ & N2 & $133.60(10)$ \\
\hline $\mathrm{O} 2$ & Np1 & $\mathrm{N} 2^{1}$ & $152.14(7)$ & & & & \\
\hline
\end{tabular}

Table S6. Bond lengths $[\AA]$ and angles $\left[{ }^{\circ}\right]$ for $\mathbf{N p}^{\mathrm{VI}} \mathbf{O}_{2} \mathbf{L}(\mathbf{M e O H})$

\begin{tabular}{|c|c|c|c|c|c|c|c|}
\hline Atom & Atom & \multicolumn{2}{|c|}{ Length/Å } & & & & \\
\hline $\mathrm{Np} 01$ & $\mathrm{O} 2$ & \multicolumn{2}{|c|}{$2.306(2)$} & $\mathrm{O} 3$ & $\mathrm{~Np} 01$ & $\mathrm{O} 5$ & $89.37(10)$ \\
\hline $\mathrm{Np} 01$ & $\mathrm{O} 3$ & \multicolumn{2}{|c|}{$1.761(2)$} & $\mathrm{O} 3$ & Np01 & N2 & $90.45(10)$ \\
\hline Np01 & $\mathrm{O} 1$ & \multicolumn{2}{|c|}{$2.225(2)$} & $\mathrm{O} 3$ & Np01 & N1 & $86.07(10)$ \\
\hline Np01 & $\mathrm{O} 4$ & \multicolumn{2}{|c|}{$1.762(2)$} & $\mathrm{O} 1$ & Np01 & $\mathrm{O} 2$ & $156.64(9)$ \\
\hline Np01 & O5 & \multicolumn{2}{|c|}{$2.447(2)$} & $\mathrm{O} 1$ & Np01 & $\mathrm{O} 5$ & $83.12(9)$ \\
\hline $\mathrm{Np} 01$ & N2 & \multicolumn{2}{|c|}{$2.546(3)$} & $\mathrm{O} 1$ & Np01 & $\mathrm{N} 2$ & $133.59(9)$ \\
\hline \multirow[t]{2}{*}{$\mathrm{Np} 01$} & N1 & \multirow{2}{*}{\multicolumn{2}{|c|}{$2.513(3)$}} & $\mathrm{O} 1$ & Np01 & N1 & $70.46(9)$ \\
\hline & & & & $\mathrm{O} 4$ & Np01 & $\mathrm{O} 2$ & $90.74(10)$ \\
\hline Atom & Atom & Atom & Angle/ ${ }^{\circ}$ & O4 & Np01 & $\mathrm{O} 1$ & $89.79(10)$ \\
\hline $\mathrm{O} 2$ & Np01 & $\mathrm{O} 5$ & $73.53(8)$ & O4 & Np01 & O5 & $89.85(10)$ \\
\hline $\mathrm{O} 2$ & Np01 & N2 & $69.76(9)$ & O4 & Np01 & $\mathrm{N} 2$ & $90.17(10)$ \\
\hline $\mathrm{O} 2$ & Np01 & N1 & $132.72(9)$ & O4 & Np01 & N1 & $94.64(10)$ \\
\hline $\mathrm{O} 3$ & Np01 & $\mathrm{O} 2$ & $89.02(10)$ & $\mathrm{O} 5$ & Np01 & $\mathrm{N} 2$ & $143.29(8)$ \\
\hline $\mathrm{O} 3$ & Np01 & $\mathrm{O} 1$ & $90.13(10)$ & O5 & Np01 & N1 & $153.15(9)$ \\
\hline $\mathrm{O} 3$ & Np01 & $\mathrm{O} 4$ & $179.22(11)$ & N1 & $\mathrm{Np} 01$ & $\mathrm{~N} 2$ & $63.30(9)$ \\
\hline
\end{tabular}




\section{References}

(1) Elgrishi, N.; J. Rountree, K.; D. McCarthy, B.; S. Rountree, E.; T. Eisenhart, T.; L. Dempsey, J. A Practical Beginner's Guide to Cyclic Voltammetry. J. Chem. Educ. 2017, 95, 197-206.

(2) Randviir, E. P. A Cross Examination of Electron Transfer Rate Constants for Carbon Screen-Printed Electrodes Using Electrochemical Impedance Spectroscopy and Cyclic Voltammetry. Electrochim. Acta. 2018, 286, 179-186.

(3) Lavagnini, I.; Antiochia, R.; Magno, F. An Extended Method for the Practical Evaluation of the Standard Rate Constant from Cyclic Voltammetric Data. Electroanalysis 2004, 16, 505-506. 